\title{
16. L'impact du contexte sur l'interprétation des composés. Le cas des composés NN en suédois et des constructions correspondantes en français
}

\author{
Maria Rosenberg \\ Stockholms universitet
}

\section{Introduction}

Cette étude contrastive focalise sur le rôle joué par le contexte en ce qui concerne l'interprétation des composés NN suédois et leurs constructions françaises qui y correspondent. Cysouw \& Wälchli (dir.) (2007), qui signalent l'utilité du corpus parallèle pour les recherches typologiques et morphologiques, observent justement que le rôle du contexte est souvent négligé dans de telles études. Elle fait partie d'un projet de recherche ayant pour but d'examiner les composés $\mathrm{NN}$ en suédois et leurs constructions correspondantes en français, en se basant sur un corpus parallèle de traduction.

L'objectif principal est donc d'examiner l'impact du contexte sur l'interprétation des composés NN en suédois. Mon hypothèse émise est que l'interprétation d'un composé NN suédois poserait, dans certains cas, des problèmes pour le traducteur, ce qui se reflète dans la traduction française. La méthode utilisée pour essayer de répondre à la question d'une influence éventuelle du contexte consiste à examiner tous les composés NN suédois attestés dans le corpus afin de juger, d'une part, s'ils sont établis ou non, et d'autre part, s'ils peuvent recevoir une interprétation ambiguë ou non hors contexte.

Le contenu de ce travail est organisé de la manière suivante. La section 2 présente le corpus parallèle et quelques données que j'ai pu en extraire. La section 3 traite du contexte ainsi que du Principe de compositionnalité et leur impact sur l'interprétation des composés. La section 4 contient l'analyse de l'impact du contexte sur l'interprétation de

Comment citer ce chapitre :

Rosenberg, Maria, L'impact du contexte sur l'interprétation des composés. Le cas des composés NN en suédois et des constructions correspondantes en français. In: Engwall, Gunnel \& Fant, Lars (eds.) Festival Romanistica. Contribuciones lingüísticas Contributions linguistiques - Contributi linguistici - Contribuições linguísticas. Stockholm Studies in Romance Languages. Stockholm: Stockholm University Press. 20I 5, pp. 3 I7-333. DOI: http://dx.doi.org/IO.I6993/bac.p. License: CC-BY 
composés NN attestés dans le corpus. Enfin, la conclusion du travail est donnée dans la section 5 .

\section{Données}

Les données dérivent d'un corpus parallèle de traduction, bidirectionnel : le français et le suédois constituent aussi bien la langue source que la langue cible. Le corpus a été compilé dans le but d'essayer de respecter deux facteurs, importants pour sa représentativité, à savoir équilibre et sélection (McEnery et al. 2006). Il représente deux types de genre textuel : textes de fiction et de non-fiction en suédois et en français, avec leurs traductions parallèles dans l'autre langue. Tous les textes inclus sont contemporains, publiés après I985, et produits par des locuteurs natifs. Les auteurs et les traducteurs des textes ont été sélectionnés afin d'avoir une grande diversité.

Cette étude se base sur les 2800 premiers mots dans les versions suédoises de chaque œuvre. La raison pour laquelle a été fixée une limite de mots-occurrences est de pouvoir comparer le nombre de mots-occurrences dans les textes source suédois à celui des textes source français, et aussi à celui des textes cible.

Tableau 1. La distribution des mots-occurrences dans le corpus parallèle

\begin{tabular}{lrlr}
\hline Suédois & Mots-occurrences & Français & Mots-occurrences \\
\hline Textes source : & 28000 & Textes cible : & 33932 \\
5 fiction + & $(23,4 \%)$ & 5 fiction + & $(28,3 \%)$ \\
5 non-fiction & & 5 non-fiction & \\
Textes cible : & 28000 & Textes source : & 2977 I \\
5 fiction + & $(23,4 \%)$ & 5 fiction + & $(24,9 \%)$ \\
5 non-fiction & & 5 non-fiction & \\
Textes source & 56000 & Textes source & 63703 \\
et cible & $(47 \%)$ & et cible & $(53 \%)$ \\
\hline Mots-occurrences totaux & & & I I9 703 (I00\%) \\
\hline
\end{tabular}

On peut constater que, pour ce qui est de la partie française du corpus, les textes cible contiennent un nombre plus élevé de mots que font les textes source. De plus, il se dégage du tableau I que les mots-occurrences 
français constituent $53 \%$ de tous les mots-occurrences dans le corpus, alors que les mots-occurrences suédois constituent $47 \%$. Les textes français contiennent donc plus de mots, comparés aux textes suédois (cf. Eriksson I997).

Regardons ensuite la distribution des composés NN dans différentes parties du corpus parallèle en suédois. De prime abord, remarquons qu'au total environ mille sept cent composés NN suédois sont attestés, constituant un peu plus de $3 \%$ de tous les mots-occurrences dans les textes suédois. En plus, les données dans le tableau 2 montrent que le type de genre textuel semble être lié à la fréquence des composés NN en suédois : les textes de non-fiction comportent plus de composés NN suédois que ceux de fiction. Ce fait pourrait, selon moi, s'expliquer par le besoin des textes de non-fiction, scientifiques, de se servir de termes plus exacts et définitoires.

Tableau 2. Composés NN dans les textes suédois de fiction et nonfiction du corpus parallèle

\begin{tabular}{lrr}
\hline Suédois & Composés NN & Fréquence relative \\
\hline Textes de fiction & 695 & $2,5 \%$ \\
Textes de non-fiction & I 046 & $3,7 \%$ \\
Tous textes & I $74 \mathrm{I}$ & $3, \mathbf{I} \%$ \\
\hline
\end{tabular}

Si on compare les textes source avec les textes cible, une autre différence se dégage aussi. Il ressort du tableau 3 qu'il y a plus de composés NN suédois dans les textes source que dans les textes cible. Les textes originaux suédois semblent constituer un environnement plus favorable pour les composés $\mathrm{NN}$, comparé aux textes suédois traduits du français. Ce fait indique qu'il y a des effets d'interférence entre les deux langues, même au niveau des mots.

Tableau 3. Composés NN dans les textes suédois source et cible du corpus parallèle

\begin{tabular}{lrr}
\hline Suédois & Composés NN & Fréquence relative \\
\hline Textes source & I 027 & $3,7 \%$ \\
Textes cible & $7 \mathrm{I} 4$ & $2,6 \%$ \\
Tous textes & I $74 \mathrm{I}$ & $3, \mathrm{I} \%$ \\
\hline
\end{tabular}


Consultons finalement la figure I, qui présente les données du corpus parallèle en focalisant sur la structure formelle des constructions françaises qui correspondent aux composés NN suédois. Il s’y dégage par exemple que le français n'utilise pas autant de composés NN que le suédois. Que la composition NN soit productive en suédois ( $c f$. Josefsson 2005) et en français ( $c f$. Fradin 2009) ne veut ainsi pas dire la même chose, en prenant en compte la fréquence relative. En revanche, le français préfère des syntagmes syntaxiques ou des constructions plus ou moins lexicalisées, pour exprimer un concept complexe là où le suédois utilise un composé $\mathrm{NN}$, comme constaté par nombreux chercheurs ( $c f$. Booij 2009; Bücking 2009; Van Goethem 2009). Or, pour nuancer ce qui vient d'être dit, mes données mettent en avant que, le plus souvent, un composé $\mathrm{NN}$ suédois correspond à un $\mathrm{N}$ en français, même si les séquences $\mathrm{N}$ de $\mathrm{N}, \mathrm{N}$ A et $\mathrm{N}$ de Dét $\mathrm{N}$ sont aussi parmi les plus fréquentes. Il y a aussi bien d'autres constructions différentes, utilisées là où le suédois a un composé $\mathrm{NN}$, comme illustré dans la figure I.

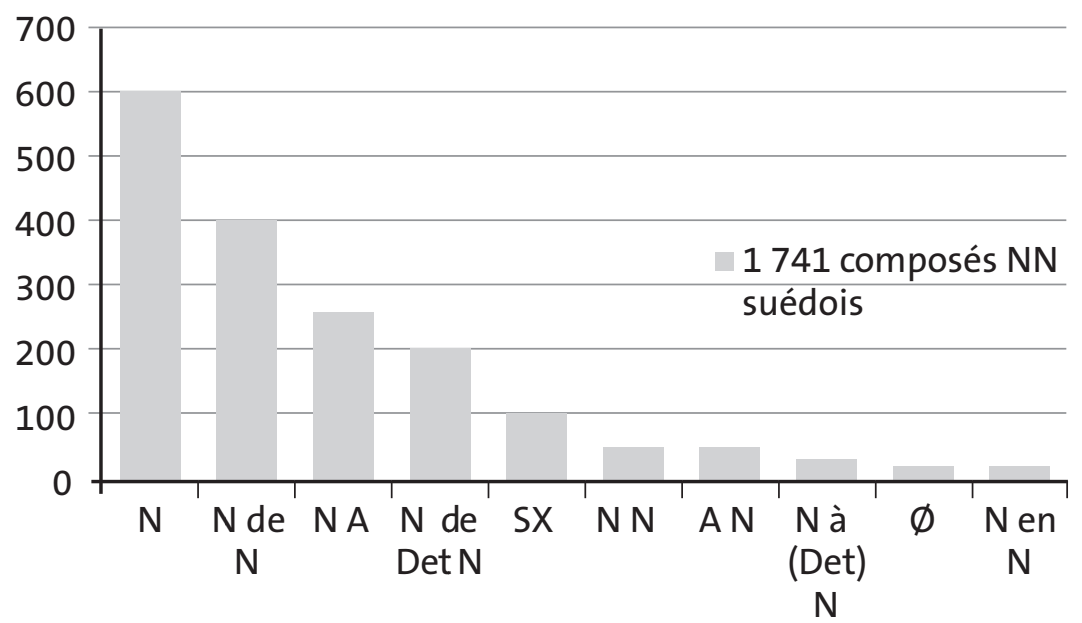

Figure 1. Unités françaises correspondant aux composés NN suédois dans le corpus parallèle

La multitude de constructions françaises correspondant à un composé NN suédois peut certainement être liée à la flexibilité sémantique manifestée par cette dernière langue. La relation sémantique interne des composés NN a été étudiée, entre autres, par Downing (I977), Levi (1978), Finin (I980) (cf. 3.I ci-dessous), et plus récemment par Jackendoff (2009), mais ne fait aucun objet de la présente étude, qui choisit de se concentrer sur l'importance du contexte pour l'interprétation des composés NN suédois. 


\section{Le rôle joué par le contexte pour l'interprétation des composés}

Dans ce qui suit seront discutés d'abord la notion du contexte et sa validité pour l'interprétation des composés NN et ensuite le Principe de compositionnalité, qui, lui aussi, est important pour l'interprétation des composés. Il serait certainement possible d'associer à cette étude aussi le concept de "vague " ou de "floue » en linguistique, et également de pousser encore plus loin la réflexion sur l'ambiguïté ${ }^{\mathrm{T}}$. Or, ceci mènerait à une étude plus étendue que ne le permet l'espace disponible ici.

\subsection{Le contexte}

Dans une perspective linguistique sur la traduction, Catford (I965) fait remarquer la différence entre contexte et co-texte :

By context we mean 'context of situation', i.e. those elements of the extratextual situation which are related to the text as being linguistically relevant: hence contextual. By co-text we mean items in the text which accompany the item under discussion: hence co-textual (1965:31).

Lorsque cette étude parle du contexte, on entendra le plus souvent le co-texte.

Finin (I980) s'intéresse au problème posé par un composé NN, étant donné que la relation sémantique entre les deux concepts ou mots entrant dans le composé n'est pas explicite. Selon lui (Finin I980: 3 I0), la sélection de la relation la plus adéquate peut dépendre d'une foule de facteurs sémantiques, pragmatiques et contextuels. Lapata (2002) souligne également trois problèmes que pose un composé pour l'interprétation automatique : (i) la haute productivité implique l'existence d'un besoin d'interpréter des formations qui ne sont pas encore établies ; (ii) la relation sémantique entre les constituants d'un composé est souvent implicite ; (iii) le contexte et des facteurs pragmatiques ont un impact sur l'interprétation.

Aussi bien Finin (1980) que Lapata (2002), se situant les deux dans un cadre TAL, signalent ainsi l'importance des facteurs contextuels pour l'interprétation d'un composé et soulignent aussi que la relation sémantique tenant entre ses constituants peut être imprécise ou flexible. Selon Gagné et al. (2005), l'interprétation des composés non-établis dépendrait le plus du contexte (ou co-texte), alors que celle des composés établis dépendrait le plus de la signification déjà établie. Ó Séaghdha $\&$ Copestake (2007) discernent quatre types de contexte (ou co-texte) qui 
sont pertinents pour l'interprétation d'un composé, si on se place dans la linguistique de corpus :

(i) les contextes du composé-type (type similarity)

(ii) les contextes des occurrences de chaque constituant (word similarity)

(iii) les contextes où se trouvent les deux constituants ensemble (relation similarity)

(iv) le contexte du composé-occurrence (token similarity).

Puisque l'interprétation d'un composé-type peut varier selon les différents contextes, ou co-textes, le type (iv) sera pertinent pour la présente étude.

Les considérations précédentes laisseraient penser, entre autres, que l'interprétation d'un composé $\mathrm{NN}$ suédois pourrait poser problème pour un traducteur qui doit rendre le composé en français. Cette idée est justement celle supposée dans mon hypothèse ( $c f$. section I). Cependant, Isabelle (I984) fait remarquer qu'il est rare qu'un composé ait une interprétation inhabituelle ou bizarre. Dans le même ordre d'idées, selon Meyer (I993), qui a examiné des composés NN allemands nouveaux, tirés des journaux quotidiens, la plupart des composés NN non-établis sont faciles à interpréter sans recourir au contexte. Ryder (I994) exprime l'avis que c'est par l'inter-médiaire de la connaissance des patrons sémantiques productifs, c'est-à-dire des relations sémantiques productives, que les composés sont créés et interprétés.

En somme, on peut donc constater qu'il semble exister deux points de vue opposés en ce qui concerne l'interprétation d'un composé NN : soit le contexte a de l'importance pour son interprétation ; soit le contexte n'affecte pas son interprétation d'une manière significative. Les deux points de vue sont cependant compatibles avec le Principe de compositionnalité, comme nous allons le voir ci-dessous.

\subsection{Le principe de compositionnalité}

Le Principe de compositionnalité stipule que

le sens d'une expression composée ne dépend que du sens de ses composants et des règles syntaxiques par lesquelles ils sont combinés (Tellier I999 : 109).

Puisque la présente étude traite des formations morphologiques sous forme de composés $\mathrm{NN}$, il faudrait plutôt utiliser " règles morphologiques ou lexicales » au lieu de "règles syntaxiques » dans la stipulation ci-dessus. 
Dans le but de garder le Principe de compositionnalité, Pelletier (2003) propose que l'interprétation d'un SN donne lieu à des valeurs sémantiques distinctes dans des contextes différents. Selon Pelletier (ibid.), l'ambiguïté contextuelle ne peut pas appartenir au sens lexical d'un $\mathrm{N}$ : les sens des constituants d'un SN restent les mêmes d'une phrase à l'autre, mais l'interprétation du SN varie.

Weiskopf (2007) estime aussi que la langue est compositionnelle : les composés NN contiennent des traits contextuels cachés, autorisant des relations différentes qui ne sont pas exprimées dans le lexique. Selon lui, le fait que les composés aient un sens sous-spécifié dans le lexique, préserve l'équilibre entre le cas où l'interprétation d'un composé NN dépend du contexte, et celui où l'interprétation d'un composé NN peut se faire sans recourir au contexte.

L'approche de Pelletier (2003) est ainsi différente de l'approche de Weiskopf (2007). J'interprète la première comme présupposant qu'un $\mathrm{N}$ homonyme (par exemple un composé) donne lieu à des entrées différentes dans le lexique, alors que selon la deuxième approche, il n'y aurait qu'une entrée lexicale, sémantiquement sous-spécifiée. Le Principe de compositionnalité est certainement pertinent pour la présente étude, puisque il s'agit d'une composition nominale, combinée à partir de deux noms. Quant à la question de savoir si un composé NN ambigu ou homonyme aurait soit une entrée lexicale sémantiquement sous-spécifiée, soit des entrées lexicales différentes, elle n'a pas trop de pertinence pour la présente étude. Car, en attestant un composé ambigu dans le corpus, il sera de toute façon nécessaire d'aller au contexte pour résoudre l'ambiguité et vérifier son interprétation. Néanmoins, cette question sera abordée dans mon travail.

Wisniewski \& Wu (20I2 : 403) avancent que la compositionnalité mène à la productivité - vu que la combinaison des concepts crée des concepts complexes qui s'accompagnent par la production d'expressions nouvelles - ainsi qu'à la systématique - étant donné qu'il nous est possible de prévoir des patrons sémantiques.

Finalement, notons qu'en règle générale, si on estime que les expressions complexes sont interprétées par des règles compositionnelles, le contexte n'aurait pas beaucoup à faire avec leur interprétation. Cependant, Recanati (20I2) émet l'opinion qu'on peut garder le Principe de compositionnalité sans oublier que le contexte joue un rôle important pour l'interprétation des expressions complexes (ce qu'estiment aussi, bien que de manière différente, Pelletier 2003 et Weiskopf 2007). 


\section{Analyse de l'impact du contexte sur l'interprétation des composés NN suédois}

Précisons d'abord que cette analyse est surtout qualitative, étant donné que le nombre d'attestations des composés non-établis ou ambigus était étonnamment faible. Elle est basée sur l'introspection, le $S A O B$ et le $S A O L$ (deux dictionnaires de l'Académie suédoise) ${ }^{2}$, mais aussi sur des recherches sur Google. Constatons ensuite que parmi tous les I 74I composés NN attestés dans le corpus, uniquement 78 (4,5\% de tous les composés $\mathrm{NN}$ ) sont estimés comme soit non-établis soit ambigus quant à leur interprétation, ce que montre le tableau 4 suivant. Quant à la question de décider si un composé est établi ou non, j'ai consulté le $S A O B$ et le $S A O L$ et aussi fait des recherches sur Google : s'il y avait moins de cinq attestations différentes sur Google, le composé était, dans presque tous les cas, jugé non-établi. Pour ce qui est de l'évaluation de l'ambiguité interprétative des composés, je me suis basée sur l'introspection en combinaison avec le $S A O B$, le $S A O L$ et des recherches googliennes ; ces dernières ont été effectuées dans le but de contrôler si mon intuition pourrait se confirmer sur la Toile.

Tableau 4. Composés NN suédois dans le corpus parallèle, jugés non-établis ou interprétativement ambigus

\begin{tabular}{lrr}
\hline Non-établis ou ambigus & 78 & $4,5 \%$ \\
\hline Établis & I 663 & $95,5 \%$ \\
Composés NN suédois & I $74 \mathrm{I}$ & I00\% \\
\hline
\end{tabular}

Dans ce qui suit seront présentés et discutés cinq cas qui sont discernés parmi les composés non-établis ou ambigus attestés dans le corpus. Il est à noter que les exemples commencent par l'attestation dans le texte source, suivie par sa traduction dans l'autre langue.

\subsection{Composés non-établis interprétables hors contexte}

Le premier cas est le plus fréquent, à savoir que la plupart des composés NN suédois non-établis attestés dans mes données sont faciles à interpréter sans recourir au contexte. Mon étude parle de la sorte en faveur à la fois des opinions exprimées par Isabelle (I984) et Meyer (I993) (cf. section 3.I), et du Principe de compositionnalité (cf. section 3.2). En revanche, elle ne semble pas confirmer l'hypothèse émise ( $c f$. section I), 
à savoir que l'interprétation d'un composé NN suédois pourrait être problématique au niveau de la traduction française. À mon avis, des composés comme les suivants dans $(\mathrm{I}-4)$ s'interprètent aussi facilement que les constructions françaises qui y correspondent :

(I) barre énergétique $v s$ energichokladkaka 'énergie-chocolat-barre'

(2) ce livre m'aura posé beaucoup de problèmes, en raison de l'énorme masse documentaire à saisir $v s$ dokumentationsmassa 'documentationmasse'

(3) hjortronkrukan 'mûre polaire-la jarre' vs Le grand tonneau de lard salé est roulé au-dehors, la caque de harengs, la caisse de pommes de terre, la jarre de mûres polaires

(4) un exercice de la paternité vs ett fadersagerande

\subsection{L'ambiguïté ressortant d'un des constituants du composé}

Dressler (2006 : 4I) fait la distinction entre quatre degrés de transparence morphosémantique à l'intérieur des composés : (i) les deux constituants sont transparents (doorbell) ; (ii) la tête est transparente, l'autre constituant est opaque (strawberry); (iii) le constituant non-tête est transparent, la tête est opaque (jailbird) ; (iv) les deux constituants sont opaques (bum-bug). La transparence du constituant tête est la plus importante pour l'interprétation.

Pour ce qui est des composés considérés susceptibles d'une interprétation ambiguë dans mes données, on peut constater que celle-ci dépend le plus souvent de l'ambiguïté lexicale d'un des constituants. Elle est facilement résolue en recourant au contexte dans lequel se trouve le composé en question. Dans l'exemple (5), le contexte est celui du prix Nobel. Aussi bien le $\mathrm{N}$ suédois, pris, que le $\mathrm{N}$ français, prix, sont ambigus entre le sens de 'récompense' et celui de 'valeur monétaire'; or dans ce contexte, la première interprétation est celle qui doit être choisie ici. Notons en plus que ce composé NN suédois est rendu par trois traductions différentes en français. Dans le composé suédois en (6), le deuxième nom, planet 'le plan', est dans sa forme définie : il consiste en un $\mathrm{N}$, plan, et une flexion définie, -et, suffixée. Par conséquent, cette forme du $\mathrm{N}$ le rend homonyme en suédois du $\mathrm{N}$ planet 'planète', ce qui avait donné une autre interprétation à ce composé (pensons par ex. à Le Petit Prince). Pour l'exemple (7) ci-dessous, le contexte est la grande chaîne de la nature : le texte parle de Linné. Le premier $\mathrm{N}$ dans le composé, övergång, doit conséquemment s'interpréter comme 'transition'. Mais, si on pense à la fameuse photo des Beatles traversant Abbey road, ce $\mathrm{N}$ peut recevoir une autre interprétation, à savoir 'passage piéton', ce 
qui change l'interprétation du composé. En faisant des recherches sur Google, j'ai pu vérifier cette deuxième interprétation. On peut y trouver plusieurs photos sur ce thème, montrant des personnes moins célèbres traversant des passages piéton un peu partout dans le monde. Dans l'exemple (8), la tête du composé, -benen 'les jambes', est ambiguë entre 'jambes d'un être humain' ou, par métonymie, 'jambes d'un pantalon', de sorte que l'interprétation du composé devient ambiguë.

(5) prisbeslut 'prix-décision' vs décision pour le prix, décision d'un prix pris/prix 'valeur monétaire' ou 'récompense' [contexte Nobel]

(6) il vend ce que d'autres ont produit, il est dès lors à l'étage des marchands $v s$ köpmannaplanet ' le marchand-plan'

planet 'le plan' ou 'planète'

[contexte économique]

(7) övergångsmänniskor 'transition-humains' vs Linné se mit en quête, par ses lectures et auprès de ses collègues, d'êtres humains intermédiaires övergång 'transition' ou 'passage piéton' [contexte histoire naturelle]

(8) Elle était déjà derrière le paravent en train de se battre avec les jambes de son jean $v s$ jeansbenen 'les jean-jambes'

\subsection{L'interprétation de la traduction française diffère de celle du composé suédois}

Il arrive dans certains cas attestés, peu nombreux, que les traductions françaises des composés NN suédois imposent une interprétation qui diffère de l'interprétation du composé suédois. À titre d'exemple, le premier nom du composé suédois donné dans (9, ci-dessous), flytt 'déménagement' ou 'déplacement', est un terme d'un sens assez général. Par conséquent, le composé suédois est assez vague quant à son interprétation, alors que la traduction française donne une interprétation plus spécifique, notamment celle d'émigrer'. En recourant au contexte, on peut facilement constater que la traduction française est justifiée, car il est question de l'émigration wallonne vers la Suède. Dans l'exemple (Io), la traduction française du composé suédois ungdomsfest, par rapport à midsommar 'la fête de la Saint-Jean', est un des rares exemples de ce qui aurait pu constituer un problème d'interprétation de la part du traducteur. Selon le contexte, il fallait plutôt dire 'fête pour les jeunes/des jeunes'. L'exemple (II) pourrait être du même genre : il y est question, à mon avis, de galettes de pain qui sont (devenues) dures ou sèches et non pas, comme dans la 
traduction, des galettes de pain dur. Dans l'exemple (I 2), l'interprétation du composé NN suédois est ambiguë d'une manière subtile en ce que le composé peut désigner soit un plateau destiné à servir le petit-déjeuner soit un plateau (quelconque) sur lequel le petit déjeuner est servi. Cette dernière interprétation est celle donnée par la traduction française. Le dernier exemple (I3) ci-dessous peut être considéré comme une traduction fautive, mais dont le traducteur est peut-être conscient ${ }^{3}$. Toutefois, cette traduction est tout à fait compréhensible, d'après moi, étant donné qu'il fait normalement plus chaud dans l'après-midi que pendant le matin.

(9) flyttförbud 'déménagement-interdiction' vs interdiction d'émigrer

(Io) ungdomsfest 'jeune-fête' vs fête de la jeunesse

(I I) de hårda brödkakorna 'les galettes de pain dures/sèches' vs les galettes de pain dur

(I2) en frukostbricka 'un petit déjeuner-plateau' vs un plateau avec le petit déjeuner

(I3) förmiddagshettan 'avant-midi-chaleur' vs la chaleur de l'après-midi

\subsection{Composés avec un sens propre et un sens figuré}

Il n'y a qu'un seul composé NN suédois attesté qui ait en même temps un sens concret ou propre et un sens abstrait ou figuré. Il est donné dans (I4). En suédois contemporain, c'est le sens abstrait qui est le plus courant, d'après moi. En lisant ce composé hors contexte, il est ainsi difficile de savoir quelle interprétation lui donner. Par conséquent, il faut recourir au contexte pour résoudre cette ambiguïté. Pourtant, la phrase originale française montre clairement qu'il est question du sens concret chez le composé suédois :

(I4) La voiture devenait elle aussi irréelle à mesure qu'il progressait. Il allait quitter les traces des pneus $v s$ spåren efter bilringarna 'les traces des pneus' [bilring 'pneu d'automobile' ou 'poignée d'amour']

En prenant en considération la validité du Principe de compositionnalité et les deux idées différentes exprimées par Pelletier (2003) et Weiskopf (2007) (cf. section 3.2), cette ambiguïté pourrait être une question de comment on conçoit l'organisation du lexique. Dans le lexique, il y a soit deux entrées différentes pour le composé bilring en suédois, soit il y a une seule entrée dans laquelle le sens du composé est laissé sous-spécifié. Toutefois, pour l'auteur qui choisit d'utiliser ce composé, l'ambiguïté n'existe point, non plus pour le traducteur, qui a tout le contexte à sa portée. 


\subsection{Ancrage contextuel facilitant l'interprétation du composé NN}

J'ai aussi pu attester quelques cas comme celui de l'exemple (I 5 ), illustrant que le contexte peut permettre la formation d'un composé nouveau, non-établi. Nous voyons que l'interprétation du composé ne pose pas de problème puisqu'il y a deux expressions précédentes auxquelles le composé, anaphorique, se réfère. Ici, on a donc affaire à un composé qui tire son origine du contexte. Notons que la traduction française utilise deux expressions quasiment identiques afin de référer à l'unité lexicale en question.

( I 5) en cigarr (första cigarren efter frukost) ... frukostcigarren 'petit déjeuner-cigare' $v s$ un cigare [...] (premier cigare après le petit déjeuner) ... le cigare d'après le petit déjeuner

\section{Conclusion}

Cette étude semble indiquer que, dans le corpus parallèle, il y a en effet très peu de composés NN suédois jugés non-établis ou ambigus quant à leur interprétation. Pourtant, parmi ceux-ci, deux cas types sont discernés : premièrement, la majorité des composés non-établis sont facilement interprétés hors contexte ; deuxièmement, l'ambiguïté d'un composé dépend le plus souvent d'une ambiguité d'un de ses constituants, laquelle est résolue en recourant au contexte. Pour le premier cas, le Principe de compositionnalité reste valable. Ce principe peut aussi être gardé pour ce qui est du deuxième cas, à mon avis, car il n'est pas forcément question de l'ambiguïté au niveau du lexique, c'est seulement au niveau interprétatif, qu'il y a une ambiguïté ( $c f$. Pelletier 2003).

Puisque le contexte ne semble pas avoir d'impact significatif sur l'interprétation de la plupart des composés $\mathrm{NN}$, qui, au contraire, sont facilement interprétables hors contexte, la conclusion qui s'impose est que l'hypothèse ne peut se confirmer. Cela veut ainsi dire que l'interprétation d'un composé NN suédois ne semble pas poser de problèmes qui se reflètent dans la traduction française. Dans cette perspective, mon résultat est un résultat nul. Pour autant, il n'est pas sans intérêt, étant donné qu'il semble indiquer qu'il faut peut-être mettre en question les résultats obtenus par exemple par Gagné et al. (2005), c'est-à-dire que l'interprétation des composés non-établis serait fortement dépendante du contexte. Plus important, mon résultat semble confirmer la validité du Principe de compositionnalité pour ce qui est de la composition NN suédoise, puisqu'en liant ce principe à l'interprétation des composés, il semble s'appliquer dans presque tous les cas. 
Quant à la question de l'organisation du lexique, cette étude ne fournit aucune réponse claire. On pourrait soit supposer qu'il y a deux ou plusieurs entrées lexicales pour un composé NN ambigu, soit estimer qu'il y a une seule entrée pour un composé NN ambigu dans laquelle son sens est sous-spécifié. Selon moi, la première option semble plus plausible en partant de l'idée que les composés sont formés par la morphologie : le sens de chacun des noms qui entrent dans le composé doit être fixé préalablement à l'application de la règle morphologique. Le fait de stipuler qu'il existe plusieurs entrées pour un composé ambigu ne concerne donc que les composés dont un des constituants est d'une interprétation ambiguë. En revanche, en ce qui concerne l'exemple (I4) ci-dessus, bilring 'pneu' ou 'poignée d'amour', il est différent, puisque dans ce cas, c'est le sens compositionnel du composé qui est ambigu entre un sens concret ou propre et un sens abstrait ou figuré. Ici, il faut plutôt considérer que c'est le sens concret qui, le plus naturellement, est à la base de l'autre. Reste à savoir s'il y a une ou deux entrées lexicales, reliées entre elles, pour un tel cas. Il me semble pourtant plus plausible qu'il serait question de deux entrées.

Finalement, il importe de souligner que, puisque cette étude est basée sur un corpus parallèle, limité entre autres par son choix de textes, une étude basée par exemple sur un corpus de quotidiens suédois aurait pu donner un tout autre résultat. Or, de mon point de vue et en m'appuyant sur l'étude de Meyer (I993), les résultats n'auraient pas été trop déviants de ceux présentés ici; toutefois ceci reste à vérifier.

\section{Notes}

I. Je tiens ici à exprimer ma reconnaissance à l'examen anonyme par les pairs pour leurs commentaires. Il convient de même de signaler que les erreurs restantes dans ce texte sont évidemment de ma responsabilité.

2. Ces deux dictionnaires sont accessibles en ligne : www.saob.se et www.saol.se.

3. Soulignons que cette étude n'a pas pour but de traiter de l'intention éventuelle du traducteur, mais aborde uniquement le résultat final de la traduction.

\section{Références}

\section{Corpus parallèle}

Allén, Sture \& Kjell Espmark. 2008. Nobelpriset i litteratur. En introduktion av Sture Allén och Kjell Espmark. Stockholm : Svenska Akademien genom Norstedts. 
Allén, Sture \& Kjell Espmark. 2008. Le Prix Nobel de littérature. Une introduction par Sture Allén et Kjell Espmark. Françoise Sule (trad.). Stockholm : Svenska Akademien genom Norstedts.

Bergman, Ingmar. I991. Den goda viljan, Stockholm : Norstedts.

Bergman, Ingmar. 1992. Les meilleures intentions. Carl Gustaf Bjurström \& Lucie Albertini (trad.). Paris : Gallimard.

Bourdieu, Pierre. I998. La domination masculine. Paris : Éditions du Seuil.

Bourdieu, Pierre. 1999. Den manliga dominansen. Boel Englund (trad.). Göteborg : Daidalos.

Braudel, Fernand. I985. La dynamique du capitalisme. Paris : Les Éditions Arthaud.

Braudel, Fernand. 1988. Kapitalismens dynamik, Gunnar Sandin (trad.), Möklinta : Gidlunds bokförlag.

Broberg, Gunnar. 2006 [1994], Carl von Linné. Stockholm : Svenska institutet.

Broberg, Gunnar. 1999 [1994], Carl von Linné. Lydie Rosseau (trad.). $2^{\mathrm{e}}$ éd., Stockholm : Svenska institutet.

Carrère, Emmanuel. 2000. L'Adversaire. Paris : P.O.L.

Carrère, Emmanuel. 200I. Doktor Romand. En sann berättelse. Suzanne Ekelöf (trad.). Stockholm : Albert Bonniers Förlag.

Ekman, Kerstin. 1993. Händelser vid vatten. Stockholm : Albert Bonniers Förlag.

Ekman, Kerstin. 1995. Crimes au bord de l'eau. Marc de Gouvenain \& Lena Grumbach (trad.). Arles : Actes Sud.

Enquist, Per Olov. 1999. Livläkarens besök. Stockholm : Norstedts.

Enquist, Per Olov. 2000. Le médecin personnel du roi. Marc de Gouvenain \& Lena Grumbach (trad.). Arles : Actes Sud.

Fredriksson, Marianne. 1994. Anna, Hanna och Johanna. Stockholm : Wahlström \& Widstrand.

Fredriksson, Marianne. I999. Hanna et ses filles. Anna Gibson (trad.). Paris : Éditions Ramsay.

Froman, Ingmarie. 2000. Sverige och Belgien. La Suède et la Belgique. Brigitte Parez (trad.). Stockholm : Svenska institutet.

Gavalda, Anna. 2004. Ensemble, c'est tout. Paris : Le Dilettante.

Gavalda, Anna. 2005. Tillsammans är man mindre ensam. Maria Björkman (trad.), Stockholm : Albert Bonniers förlag. 
Houellebecq, Michel. 200I. Plateforme. Paris : Flammarion.

Houellebecq, Michel. 2002. Plattform. Anders Bodegård (trad.). Stockholm : Albert Bonniers förlag.

Johannisson, Karin. I989. Levande lärdom. Uppsala universitet under fem sekler. Uppsala : Uppsala University Press.

Johannisson, Karin. I989. Le savoir vivant. Cinq siècles d'bistoire de l'université d'Upsal. Christiane Landner (trad.). Uppsala : Uppsala University Press.

Juillard, Jacques. I994. Ce fascisme qui vient... Paris : Éditions du Seuil.

Juillard, Jacques. I994. Fascism $i$ antågande. Charlotta Levay (trad.). Tollarp : Studiekamraten.

Kristeva, Julia. I988. Étrangers à nous-mêmes. Paris : Librairie Arthème Fayard.

Kristeva, Julia. I99 . Främlingar för oss själva. Ann Runnqvist-Vinde (trad.). Stockholm : Natur och Kultur.

Larsson, Stieg. 2006. Flickan som lekte med elden. Stockholm : Norstedts.

Larsson, Stieg. 2006. La fille qui rêvait d'un bidon d'essence et d'une allumette. Lena Grumbach et Marc de Gouvenain (trad.). Arles : Actes sud.

Le Clézio, Jean-Marie Gustave. I992. Étoile errante. Paris : Gallimard.

Le Clézio, Jean-Marie Gustave. I995. Vandrande stjärna. Ulla Bruncrona (trad.). Stockholm : Norstedts.

Roudinesco, Elisabeth. I993. Jacques Lacan. Esquisse d'une vie, histoire d'un système de pensée. Paris : Librairie Arthème Fayard.

Roudinesco, Elisabeth. I994. Jacques Lacan. En levnadsteckning, ett tankesystems historia. Eva Backelin (trad.). Per Magnus Johansson, (fackgranskning och introduktion). Stockholm : Brutus Östlings Bokförlag Symposion AB.

Tidholm, PO \& Lilja, Agneta. 2004. Det ska vi fira! - svenska traditioner och högtider. http://www.sweden.se/otherlanguages/otherlanguages/otherlanguages/3738/Swedish/Det-ska-vi-fira-Svenska-traditioner-och-hogtider/ [05/04/20II].

Tidholm, PO \& Agneta Lilja. 2004. Festivités à la suédoise - traditions et fêtes, Lydie Rousseau (trad.) http://www.sweden.se/fr/Accueil/Style-de-vie/ Festivites-a-la-suedoise/ [05/04/20I I].

Tournier, Michel. I985. La goutte d'or. Paris : Gallimard.

Tournier, Michel. I987. Gulddroppen. Carl Gustaf Bjurström (trad.). Stockholm : Albert Bonniers Förlag. 


\section{Ouvrages cités}

Booij, Geert. 2009. "Compounding and Construction Morphology ». In : Lieber, Rochelle \& Pavol Štekauer (dir.), The Oxford Handbook of Compounding. Oxford: Oxford University Press. 20I-2I6.

Bücking, Sebastian. 2009. "How do Phrasal and Lexical Modification Differ? Contrasting Adjective-Noun combinations in German ». Word Structure, 2(2) : I 84-204.

Catford, John Cunnison. 1965. A Linguistic Theory of Translation. An Essay in Applied Linguistics. London : Oxford University Press.

Cysouw, Micael \& Bernhard Wälchli (dir.). (2007). " Parallel Texts. Using Translational Equivalents in Linguistic Typology ». Numéro thématique de STUF - Language Typology and Universals, 60(2).

Downing, Pamela. 1977. " On the Creation and Use of English Compound Nouns ». Language, 53(4): 810-842.

Dressler, Wolfgang U. 2006. "Compound Types ». In : Libben, Gary \& Gonia Jarema (dir.), The Representation and Processing of Compound Words. Oxford : Oxford University Press. 23-44.

Eriksson, Olof. 1997. Språk $i$ kontrast. En jämförande studie av svensk och fransk meningsstruktur. Göteborg : Akademiförlaget.

Finin, Timothy. I980. "The Semantic Interpretation of Nominal Compounds ». In : Proceedings of First Annual National Conference on Artificial Intelligence. Stanford, California. 3 I0-3 I 5.

Fradin, Bernard. 2009. "IE, Romance: French ». In : Lieber, Rochelle \& Pavoll Štekauer (dir.). The Oxford Handbook of Compounding. Oxford : Oxford University Press. 4I7-435.

Gagné, Christina, Thomas L. Spalding \& Melissa Gorrie C. 2005. «Sentential Context and the Interpretation of Familiar Open-Compounds and Novel Modifier-Noun Phrases ». Language and Speech, 48(2) : 203-22I.

Isabelle,Pierre. 1984. "Another Look at Nominal Compounds ».In:Proceedings of the Ioth International Conference on Computational Linguistics and the 22nd Annual Meeting of the Association for Computational Linguistics. Stanford CAL: Stanford University. 509-5 I6.

Jackendoff, Ray. 2009. "Compounding in the Parallel Architecture and Conceptual Semantics». In : Lieber, Rochelle \& Pavol Štekauer (dir.), The Oxford Handbook of Compounding. Oxford: Oxford University Press. IO5-I 28 .

Josefsson, Gunlög. 2005. Ord. Lund : Studentlitteratur. 
Lapata, Maria. 2002. "The Disambiguation of Nominalizations ". Computational Linguistics, 28 : 357-388.

Levi, Judith N. I978. The Syntax and Semantics of Complex Nominals. New York : Academic Press.

McEnery, Tony, Richard Xiao \& Yukio Tono. 2006. Corpus-Based Language Studies: An Advanced Resource Book. London/New York : Routledge.

Meyer, Ralf. I993. Compound Comprehension in Isolation and in Context: the Contribution of Conceptual and Discourse Knowledge to the Comprehension of German Novel Noun-Noun Compounds. Tübingen : Niemeyer.

Ó Séaghdha, Diamud \& Ann Copestake. 2007. "Co-occurrence Contexts for Noun Compound Interpretation ". Proceedings of the Workshop on A Broader perspective on Multiword Expressions. ACL 2007, Prague, Czech Republic. 57-64.

Pelletier, Francis Jeffry. 2003. "Context Dependence and Compositionality ". Mind \& Language, I8(2): I48-I6I.

Recanati, François. 20I2. "Compositionality, Flexibility, and Context Dependence ». In : Werning, Markus, Wolfram Hinzen, \& Edouard Machery (dir.). The Oxford Handbook of Compositionality. Oxford : Oxford University Press. I75-I9I.

Ryder, Mary Ellen. I994. Ordered Chaos: The Interpretation of English NounNoun Compounds. Berkeley : University of California Press.

Tellier, Isabelle. I999. "Rôle de la compositionnalité dans l'acquisition d'une



Van Goethem, Kristel. 2009. "Choosing between $\mathrm{A}+\mathrm{N}$ Compounds and Lexicalized $\mathrm{A}+\mathrm{N}$ Phrases: The Position of French in Comparison to Germanic Languages ». Word Structure, 2(2) : $24 \mathrm{I}-253$.

Weiskopf, Daniel A. 2007. " Compound Nominals, Context, and Compositionality ". Synthese, I 56. I6I-204.

Wisniewski, Edward. J. \& Jing Wu. 20I2. "Emergency !!!! Challenges to a Compositional Understanding of Noun-Noun Combinations ». In : Werning, Markus, Wolfram Hinzen, \& Edouard Machery (dir.). The Oxford Handbook of Compositionality. Oxford : Oxford University Press. 403-4 I7. 